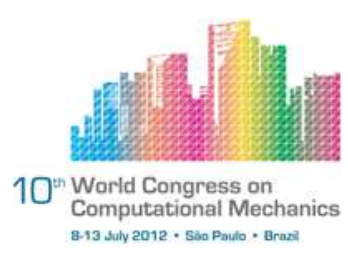

\title{
COMPARISONS BETWEEN MIXTURE THEORY AND DARCY LAW FOR TWO-PHASE FLOW IN POROUS MEDIA USING GITT AND FLUENT SIMULATIONS
}

\author{
R.A.C. Dias ${ }^{1}$, D.J.N.M. Chalhub ${ }^{2}$, T.J.L de Oliveira $^{3}$, L.A. Sphaier $^{2}$, P.D. Fernandes ${ }^{3}$ \\ ${ }^{1}$ Engineering Simulation Scientific Software (ESSS), Av. Presidente Vargas 3131, Centro
} Empresarial Cidade Nova, $12^{\circ}$ andar - 1203, Cidade Nova CEP 20210-031, Rio de Janeiro RJ - Brasil (rodrigo.dias@esss.com.br)

${ }^{2}$ Laboratório de Mecânica Teórica e Aplicada, Programa de Pós-Graduação em Engenharia Mecânica, Departamento de Engenharia Mecânica, Universidade Federal Fluminense, Rua Passo da Pátria 156, bloco E, sala 216, Niterói, RJ, 24210-240, Brazil

${ }^{3}$ Petrobras CENPES, Av. Horácio Macedo, 950 - Prédio 20 - Sala 2040, Cidade Universitária, Ilha do Fundão, Rio de Janeiro - RJ - Brasil CEP: 21948-91

\begin{abstract}
Water injection into oil formations has a very wide application in the oil industry. The injection of water has as main objective: to maintain formation pressure and provide the recovery of oil by water displacement. This work compares two different theoretical formulations used in reservoir simulations. One is the saturation differential equation and the other is the mixture theory equation applied to fluid flow in porous media. The saturation differential equation is widely used in reservoir simulations. This equation is found when Darcy's Law for each phase is applied in the continuity equation for each phase. However, there is another theoretical approach for multiphase flows in porous media. Mixture theory allows a local description of the flow in a porous medium, supported by a thermodynamically consistent theory which generalizes the classical continuum mechanics. This article compares the results obtained by the equation based on the mixture theory with the saturation differential equation. To solve the saturation equation the Generalized Integral Transform Technique (GITT) was employed. The GITT has been successfully employed in various petroleum reservoir simulation problems. The numerical method for the solution of mixture theory was obtained using advanced, commercial, general-purpose CFD code: FLUENT.
\end{abstract}

Keywords: Integral Transform Technique, Finite Volumes Method, Waterflooding, Porous Media

\section{INTRODUCTION}

Waterflooding is dominant among fluid injection methods and is without question responsible for the current high level of producing rate and reserves. Its popularity is accounted 
for by (1) the general availability of water, (2) the relative ease with which water is injected, due to the hydraulic head it possesses in the injection well (3) the ability with which water spreads through an oil-bearing formation and (4) water's efficiency in diplacing oil.

The first approach to study two-phase flow in porous media made in this article is based on the Darcy's Law. The saturation differential equation is widely used in reservoir simulations. This equation is nothing more than a rearrangement of the Black Oil model. To solve this first approach the Generalized Integral Transform Technique was employed. The Generalized Integral Transform Technique (GITT) [5] deals with expansions of the sought solution in terms of bases of infinite orthogonal eigenfunctions, maintaining the solution process always within a continuum domain. The transformation of the original problem results in a coupled system, generally composed of ordinary differential equations, which can be readily solved by well established numerical routines that allow precision control. Nevertheless, as the infinite series must be truncated so that any application can be made, a truncation error is involved. Interesting applications of the GITT in porous media problems can be seen in $[4,18,13]$.

Another approach that can be used to understand the fluid flow in porous media is based on continuum theory of mixtures, a generalization of continuum mechanics. The importance of the study of mixtures was recognized long ago and mathematical models have been developed to describe the flow of such mixtures. The pioneering works of Fick [8] and Darcy [6] deserve special mention. These early attempts were aimed at describing the diffusion or flow of one constituent of the mixture through another. While the prediction of Darcy's Law provide good agreement in many physical situations, large departures from the predictions of these laws are also possible in practice. Additionally, Darcy's Law is quite inadequate in proving some important information on diffusion processes. For instance, this law cannot predict the stresses or strain in the solid, or the flow field of the fluid. Such information is critical in many problems where the solid may fracture or rupture due to the stress or strain induced in them.

The basic premise of the mixture theory is that the space occupied by a mixture can be considered as being occupied co-jointly by the various constituents of the mixtures, each considered as a continuum in its own right. Thus, at each point in the space occupied by the mixture, there will be a particle belonging to each of the constituents. The theory of mixtures allows for the interconversion of mass between the various constituents. Balance laws for mass, linear momentum, angular momentum and energy take into account contributions of any of these quantities with regard to a particular constituent due to the influence of the other constituents. An appropriete thermomechanical setting can also be provided within which to study mixtures that take into account the basic laws of thermomechanics.

The theoretical development for studying the mechanics of mixtures started with the pioneering work of [21, 22] followed by those of [24], [23], [1], [9], [10], [19], [2] and others. Mixture theory allows a local description of the flow in a porous medium, supported by a thermodynamically consistent theory which generalizes the classical continuum mechanics [15].

The objective of this study is to compare the use of the Darcy law with the theory of mixture. The problem chosen is of great importance to the petroleum industry. the displace- 
ment of oil by water is the simplest form of oil recovery.

\section{SATURATION DIFFERENTIAL EQUATION}

Darcy's Law is extended to multiphase flow by postulating that these same phase pressures are involved in causing each fluid to flow and neglecting gravity effects [17]:

$$
\begin{aligned}
\mathbf{v}_{s w} & =-\frac{K k_{w}}{\mu_{w}} \nabla p_{w} \\
\mathbf{v}_{s o} & =-\frac{K k_{o}}{\mu_{o}} \nabla p_{o}
\end{aligned}
$$

here $\mathbf{v}_{o}$, and $\mathbf{v}_{w}$ are the superficial velocities for the oil and water, respectively, $\mu_{o}$ and $\mu_{w}$ are the respective viscosities. $k_{n}$ and $k_{w}$ are the relative permeabilities for flow for each of the two fluids and $K$ is the absolute permeability of the medium.

The mass conservation for a porous medium is given by [16]:

$$
\begin{gathered}
\frac{\partial\left(\phi \rho_{w} S_{w}\right)}{\partial t}+\nabla \cdot\left(\rho_{w} \mathbf{v}_{s w}\right)=0 \\
\frac{\partial\left(\phi \rho_{o} S_{o}\right)}{\partial t}+\nabla \cdot\left(\rho_{o} \mathbf{v}_{s o}\right)=0
\end{gathered}
$$

for incompressible fluids, negligible the capillary effects, and considering the porosity constant in time. The saturation differential equation is given by:

$$
\phi \frac{\partial S_{w}(x, t)}{\partial t}+\nabla \cdot\left(\mathbf{v}_{s t} f_{w}\left(S_{w}\right)\right)=0
$$

where $\mathbf{v}_{s t}$ is the total velocity:

$$
\mathbf{v}_{s t}=\mathbf{v}_{s w}+\mathbf{v}_{s o}
$$

or in quasilinear and one dimensional form:

$$
\phi \frac{\partial S_{w}(x, t)}{\partial t}+u_{s t} \frac{\mathrm{d} f_{w}\left(S_{w}\right)}{\mathrm{d} S_{w}} \frac{\partial S_{w}(x, t)}{\partial x}=0
$$

The above equation, known as the "Buckley-Leverett Equation" [3] is a nonlinear hyperbolic transport equation, where the coefficient multiplying the term $\partial S_{w}(x, t) / \partial x$ is a nonlinear function of $S_{w}$. This term has particularly interesting properties and allow the investigation of a series of properties of more complex flows being widely studied in the literature $[12,7]$.

\section{MIXTURE THEORY}

By the mixture theory, the mass balance may be written as:

$$
\frac{\partial \rho_{i}}{\partial t}+\nabla \cdot\left(\rho_{i} \mathbf{v}_{i}\right)=0
$$

in which $\rho_{i}$ is the ith constituent mass density in mixture, and $\mathbf{v}_{i}$ is the ith constituent velocity in mixture. The balance of linear momentum is given by:

$$
\frac{\partial\left(\rho_{i} \mathbf{v}_{i}\right)}{\partial t}+\nabla \cdot\left(\rho_{i} \mathbf{v}_{i} \mathbf{v}_{i}\right)=\nabla \cdot\left(\mathbf{T}_{i}\right)+\rho_{i} \mathbf{g}_{i}+\mathbf{m}_{i}
$$


which $\mathbf{T}_{i}$ is the partial stress tensor associated with the ith constituent, $\mathbf{g}_{i}$ is a body force per unit mass acting on the ith constituent, and $\mathbf{m}_{i}$ is an interaction force per unit volume acting on the ith constituent due to its interaction with the other constituents of the mixture.

In multiphase flows, since porosity is a parameter representing the volume blockage in porous media, all the phases in a multiphase flow system share the same porosity. Consequently, for the general porous-media modeling formulation, physical laws and governing equations are applied to the corresponding phase. The mass conservation for both phases is given by:

$$
\begin{gathered}
\frac{\partial\left(\phi \alpha_{w} \rho_{w}\right)}{\partial t}+\nabla \cdot\left(\phi \rho_{w} \alpha_{w} \mathbf{v}_{w}\right)=0 \\
\frac{\partial\left(\phi \alpha_{o} \rho_{o}\right)}{\partial t}+\nabla \cdot\left(\phi \rho_{o} \alpha_{o} \mathbf{v}_{o}\right)=0
\end{gathered}
$$

The balance of linear momentum for water is given by:

$$
\frac{\partial\left(\rho \alpha_{w} \phi \mathbf{v}_{w}\right)}{\partial t}+\nabla \cdot\left(\rho_{w} \alpha_{w} \phi \mathbf{v}_{w} \mathbf{v}_{w}\right)=-\phi \alpha_{w} \nabla p+\phi^{2} \alpha_{w} \mu \nabla^{2} \mathbf{v}_{w}-\frac{\mu \phi^{2} \alpha_{w} \mathbf{v}_{w}}{K k_{w}}+\phi \alpha_{w} \rho_{w} \mathbf{g}
$$

The balance of linear momentum for oil is written as:

$$
\frac{\partial\left(\rho \alpha_{o} \phi \mathbf{v}_{o}\right)}{\partial t}+\nabla \cdot\left(\rho_{o} \alpha_{o} \phi \mathbf{v}_{o} \mathbf{v}_{o}\right)=-\phi \alpha_{o} \nabla p+\phi^{2} \alpha_{o} \mu \nabla^{2} \mathbf{v}_{o}-\frac{\mu \phi^{2} \alpha_{o} \mathbf{v}_{o}}{K k_{o}}+\phi \alpha_{o} \rho_{o} \mathbf{g}
$$

This physical velocity formulation has been implemented in an advanced, commercial, generalpurpose CFD code, FLUENT .

\section{PROBLEM FORMULATION}

The studied problem is that of displacement of oil by water in porous media. The incompressible laminar biphase flow within a porous media is considered by two different theories. As the case study is one-dimensional, the solutions can be compared with the analytical solution of the best known two phase porous medium equation: the Buckley-Leverett equation.

A uniform mesh with one hundred elements was used in the FLUENT simulation (mixture theory). The time derivative was discretized using backward differences and firstorder implicit temporal discretization was used. The advantage of the fully implicit scheme is that it is unconditionally stable with respect to time step size. The spatial discretization was computed by the Green-Gauss Cell-Based method for gradients and First-Order Upwind Scheme for interpolating faces values in convection terms. SIMPLE was the pressure velocity coupling segregated algorithm chosen.

The interaction between the phases is given by the relative permeability curve. In this article two relative permeability curves are considered for comparison between the different approaches. A general form for these curves can be given by:

$$
k_{w}\left(S_{w}\right)=\frac{K_{r w \max }\left(S_{w}-S_{w i}\right)^{n w}}{\left(1-S_{o r}-S_{w i}\right)}
$$




$$
k_{o}\left(S_{w}\right)=\frac{K_{\text {romax }}\left(1-S_{o r}-S_{w}\right)^{n o}}{\left(1-S_{o r}-S_{w i}\right)}
$$

Another important function is the fractional flow. This function is responsible for the problem non-linearity in the saturation differential equation.

$$
f_{w}\left(S_{w}\right)=\frac{\frac{k_{w}\left(S_{w}\right)}{\mu_{w}}}{\frac{k_{w}\left(S_{w}\right)}{\mu_{w}}+\frac{k_{o}\left(S_{w}\right)}{\mu_{o}}}
$$

For all cases studied, the total velocity into the saturation differential equation is $u_{s t}=1.0 \times$ $10^{-5}$.

\subsection{Linear Permeability Curve}

The simplest case of relative permeability curves in reservoir simulation was studied. The linear relative permeability curves and the data used in this case are shown in table 1:

Table 1. Data for Simulation

\begin{tabular}{l|c|c|l} 
Properties & Values & Properties & Values \\
\hline$K_{\text {rwmax }}$ & 1 & $L$ & $1 \mathrm{~m}$ \\
\hline$K_{\text {romax }}$ & 1 & $\phi$ & 0.2 \\
\hline$S_{o r}$ & 0 & $K$ & $4.737 \times 10^{-9} \mathrm{~m}^{2}$ \\
\hline$S_{w i}$ & 0 & $\mu_{w}$ & $0.01 \mathrm{~kg} / \mathrm{ms}$ \\
\hline$n o, n w$ & 1 & $\mu_{o}$ & $0.02 \mathrm{~kg} / \mathrm{ms}$ \\
\hline
\end{tabular}

The relative permeability curves and the fractional flow function for this case is showed in Fig. 1.

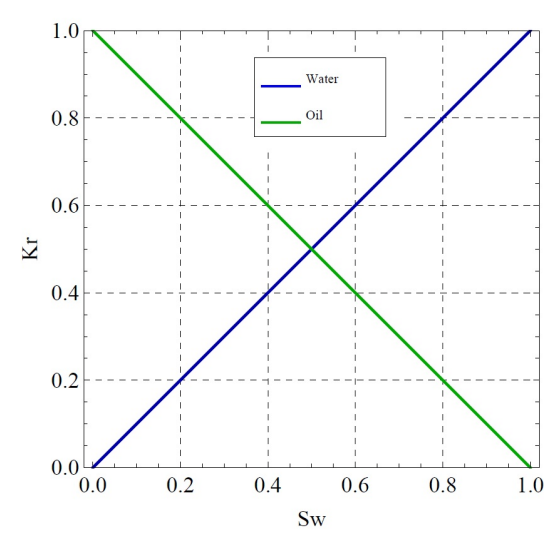

(a) Relative Permeability Curves

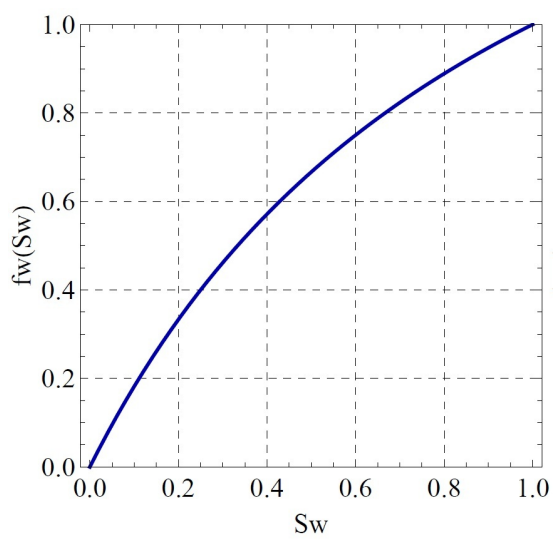

(b) Fractional Flow Function

Figure 1. Linear Permeability Curves

The boundary conditions for the present case are described below:

- For the saturation differential equation

$$
S_{w}(0, t)=1, \quad\left(\frac{\partial S_{w}}{\partial x}\right)_{x=L}=0, \quad \text { for } \quad t \geq 0
$$


- For the Mixture Theory

Inlet velocity $v(0, t)=1.0 \times 10^{-5}$ and symmetry condition at $x=L$

The initial conditions for this cases are given by:

- For the saturation differential equation

$$
S_{w}(x, 0)=S_{w i}=0,
$$

- For the Mixture Theory

Inlet velocity $v_{w}(x, 0)=0$ and $\alpha_{w}(x, 0)=S_{w i}=0$

\subsection{Non-Linear Permeability Curve}

For the second case, a model closer to reality was made. Second degree polynomials were used in relative permeability curves. This case of relative permeability curves is more common in reservoir simulation. The polynomial relative permeability curves and the data used in this case are shown in table 2 and in Fig. 2:

Table 2. Data for Simulation

\begin{tabular}{l|c|c|l} 
Properties & Values & Properties & Values \\
\hline$K_{\text {rwmax }}$ & 1 & $L$ & $1 \mathrm{~m}$ \\
\hline$K_{\text {romax }}$ & 1 & $\phi$ & 0.2 \\
\hline$S_{\text {or }}$ & 0 & $K$ & $4.737 \times 10^{-9} \mathrm{~m}^{2}$ \\
\hline$S_{w i}$ & 0 & $\mu_{w}$ & $0.01 \mathrm{~kg} / \mathrm{ms}$ \\
\hline$n o, n w$ & 2 & $\mu_{o}$ & $0.02 \mathrm{~kg} / \mathrm{ms}$ \\
\hline
\end{tabular}

The relative permeability curves and the fractional flow function for this case are showed in Fig. 2. This form of the fractional flow function is frequently found in the literature. It is intuitive to see by the charts below that the presence of more water in porous media will result in greater facility of water flow in the domain.

The boundary conditions for the present case are given by:

- For the saturation differential equation

$$
S_{w}(0, t)=0.73, \quad\left(\frac{\partial S_{w}}{\partial x}\right)_{x=L}=0, \quad \text { in } \quad t \geq 0,
$$

- For the Mixture Theory

Inlet velocity $v(0, t)=1.0 \times 10^{-5}$ and symmetry condition in $x=L$

The initial conditions for this cases are given by:

- For the saturation differential equation

$$
S_{w}(x, 0)=S_{w i}=0.16,
$$

- For the Mixture Theory

Inlet velocity $v_{w}(x, 0)=0$ and $\alpha_{w}(x, 0)=S_{w i}=0.16$ 


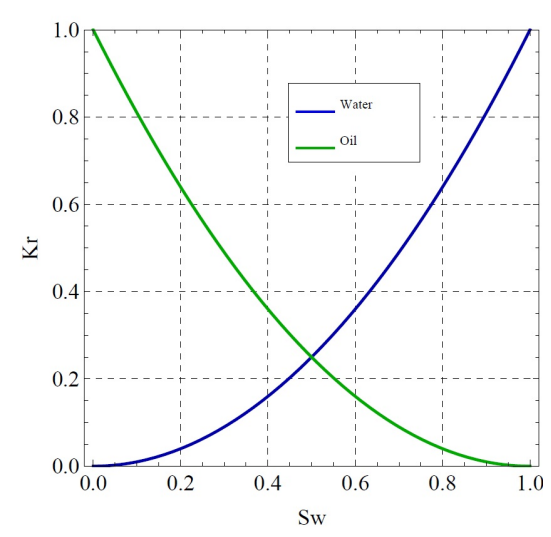

(a) Relative Permeability Curves

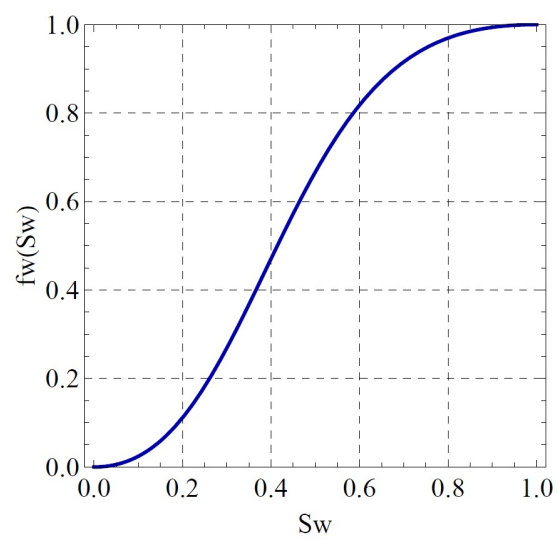

(b) Fractional Flow Function

Figure 2. Non Linear Permeability Curve

\section{INTEGRAL TRANSFORM SOLUTION}

The integral transform technique consists on seeking an eigenfunction expansion solution based on an integral transform pair. In order to obtain this eigenfunction expansion, a diffusion term is introduced to the equation, in which the $H$ parameter will be considered large.

$$
\begin{gathered}
\phi \frac{\partial S_{w}(x, t)}{\partial t}+u_{t} \frac{\mathrm{d} f_{w}\left(S_{w}\right)}{\mathrm{d} S_{w}} \frac{\partial S_{w}(x, t)}{\partial x}-\frac{1}{H} \frac{\partial^{2} S_{w}}{\partial x^{2}}=0 \\
S_{w}(0, t)=1-S_{o r} \\
S_{w}(1, t)=S_{w i r r} \\
S_{w}(x, 0)=S_{w i r r}
\end{gathered}
$$

A traditional procedure in GITT solutions is the elimination of the nonhomogeneous boundary conditions with an introduction of a filter function.

$$
S_{w}(x, t)=S_{H}(x, t)+S_{F}(x)
$$

which, for this problem, the filter function is chosen to be:

$$
S_{F}(x)=\left(S_{w i r r}-S_{w o}\right) x+S_{w o}
$$

The filter function separation provides the following filtered equation:

$$
\begin{gathered}
\frac{\partial S_{H}}{\partial t}+f_{w}^{\prime}\left(S_{w}\right)\left(\frac{\partial S_{H}}{\partial x}+\frac{\partial S_{F}}{\partial x}\right)-\frac{1}{H} \frac{\partial^{2} S_{H}}{\partial x^{2}}=F(x) \\
S_{H}(0, t)=0 \\
S_{H}(1, t)=0 \\
S_{H}(x, 0)=S_{\text {wirr }}-S_{F}(x)
\end{gathered}
$$

where

$$
F(x)=\frac{1}{H} \frac{\partial^{2} S_{F}}{\partial x^{2}}
$$


The eigenfunction expansion solution is based the following transformation pair:

$$
\begin{aligned}
\text { Inversion } & \rightarrow S_{H}(x, t)=\sum_{k=1}^{\infty} \frac{\bar{S}_{H k}(t) \Psi_{k}(x)}{N_{k}}, \\
\text { Transform } & \rightarrow \bar{S}_{H k}(t)=\int_{0}^{1} S_{H}(x, t) \Psi_{k}(x) \mathrm{d} x,
\end{aligned}
$$

where $\Psi_{x}(x)$ are orthogonal solutions of a Sturm-Liouville eigenvalue problem. Traditionally, the one-dimensional Helmholtz problem is chosen:

$$
\begin{gathered}
\Psi^{\prime \prime}(x)+\lambda^{2} \Psi(x)=0, \\
\Psi(0)=0, \\
\Psi(1)=0,
\end{gathered}
$$

The norms of the eigenfunction $\left(\Psi_{k}\right)$ are given by:

$$
N_{k}=\int_{0}^{1} \Psi_{k}^{2}(x) \mathrm{d} x
$$

These eigenvalue problems lead to infinite nontrivial solutions as shown bellow:

$$
\begin{gathered}
\Psi_{k}(x)=\sin \left(\lambda_{k} x\right) \\
\lambda_{k}=\pi k \quad \text { where } \quad k=1,2,3, \ldots
\end{gathered}
$$

Using the solution arising from the previous eigenproblem, the transformation of the original problem is carried-out by operating equation (24a) with the integral operator $\int_{0}^{1} \bullet \psi_{n}(x) \mathrm{d} x$, employing the associated boundary conditions and eigenproblem information:

The problem is transformed using the transformation operator defined in eq. (27). Almost all the terms of the equation can be directly transformed remaining two terms on the right hand side of the equation, which cannot be directly transformed.

$$
\frac{\mathrm{d} \bar{S}_{H k}}{\mathrm{~d} t}+\frac{\lambda_{k}^{2}}{H} \bar{S}_{H k}-\bar{F}_{k}=-\int_{0}^{1} \Psi_{k} f_{w}^{\prime} \frac{\partial S_{F}}{\partial x} \mathrm{~d} x-\int_{0}^{1} \Psi_{k} f_{w}^{\prime} \frac{\partial S_{H}}{\partial x} \mathrm{~d} x
$$

where

$$
\bar{F}_{k}=\int_{0}^{1} F \Psi_{k} \mathrm{~d} x
$$

In order to transform these two terms, a linear approximation of the function $f_{w}^{\prime}$ inside a subdomain $\left(x_{q-1}<x<x_{q}\right)$, in a manner that:

$$
\int_{0}^{1} f_{w}^{\prime} g\left(S_{H}, x\right) \mathrm{d} x=\sum_{q=1}^{q_{\max }} \int_{x_{q-1}}^{x_{q}}\left[a_{q}(t) x+b_{q}(t)\right] g\left(S_{H}, x\right) \mathrm{d} x
$$

where the coefficients of the linearization are:

$$
\begin{gathered}
a_{q}(t)=\frac{f_{w_{q}{ }^{\prime}}-f_{w_{q-1}}^{\prime}}{x_{q}-x_{q-1}} \\
b_{q}(t)=\frac{x_{q} f_{w_{q-1}}{ }^{\prime}-x_{q-1} f_{w_{q}}{ }^{\prime}}{x_{q}-x_{q-1}}
\end{gathered}
$$


Applying this linearization to the equation (32), the following transformed system is obtained:

$$
\begin{aligned}
\frac{\mathrm{d} \bar{S}_{H k}}{\mathrm{~d} t}+ & \frac{\lambda_{k}^{2}}{H} \bar{S}_{H k}-\frac{\bar{F}_{k}}{H}=-\sum_{q=1}^{q \max }\left(f_{w_{q}}{ }^{\prime}-f_{w_{q-1}}{ }^{\prime}\right) A_{q k}-\sum_{q=1}^{q \max }\left(x_{q} f_{w_{q-1}}{ }^{\prime}-x_{q-1} f_{w_{q}}{ }^{\prime}\right) B_{q k} \\
& -\sum_{q=1}^{q \operatorname{qmax}} \sum_{i=1}^{\infty}\left(f_{w_{q}}{ }^{\prime}-f_{w_{q-1}}{ }^{\prime}\right) C_{i q k} \bar{S}_{H i}-\sum_{q=1}^{q \max } \sum_{i=1}^{\infty}\left(x_{q} f_{w_{q-1}{ }^{\prime}}-x_{q-1} f_{w_{q}}{ }^{\prime}\right) D_{i q k} \bar{S}_{H i}
\end{aligned}
$$

where

$$
\begin{gathered}
f_{w_{q}}^{\prime}=f_{w_{q}^{\prime}}^{\prime}\left(S_{w}, x_{q}\right)=f_{w_{q}^{\prime}}^{\prime}\left(S_{H}+S_{F}, x_{q}\right)=f_{w_{q}^{\prime}}^{\prime}\left(\sum_{k=1}^{\infty} \frac{\bar{S}_{H k} \Psi_{k}}{N_{k}}+S_{F}, x_{q}\right) \\
f_{w_{q-1}}^{\prime}=f_{w_{q-1}}^{\prime}\left(S_{w}, x_{q-1}\right)=f_{w_{q-1}}^{\prime}\left(S_{H}+S_{F}, x_{q-1}\right)=f_{w_{q-1}}^{\prime}\left(\sum_{k=1}^{\infty} \frac{\bar{S}_{H k} \Psi_{k}}{N_{k}}+S_{F}, x_{q-1}\right) \\
A_{q k}=\frac{1}{x_{q}-x_{q-1}} \int_{x_{q-1}}^{x_{q}} x \frac{\mathrm{d} S_{F}}{\mathrm{~d} x} \Psi_{k} \mathrm{~d} x \\
B_{q k}=\frac{1}{x_{q}-x_{q-1}} \int_{x_{q-1}}^{x_{q}} \frac{\mathrm{d} S_{F}}{\mathrm{~d} x} \Psi_{k} \mathrm{~d} x \\
C_{i q k}=\frac{1}{N_{i}\left(x_{q}-x_{q-1}\right)} \int_{x_{q-1}}^{x_{q}} x \Psi_{k} \Psi_{i}^{\prime} \mathrm{d} x \\
D_{i q k}=\frac{1}{N_{i}\left(x_{q}-x_{q-1}\right)} \int_{x_{q-1}}^{x_{q}} \Psi_{k} \Psi_{i}^{\prime} \mathrm{d} x
\end{gathered}
$$

The transformation of the initial condition leads to:

$$
\bar{S}_{H k}(0)=\int_{0}^{1}\left(S_{w i r r}-S_{F}\right) \Psi_{k}(x) \mathrm{d} x
$$

The solution of the transformed potentials is obtained by truncating the infinite system representation (37) to a finite order and employing a commercially or publicly available dedicated ODE solver. In this work, the UNIT algorithm [20], implemented on the Mathematica software which uses the NDSolve routine was employed.

\section{RESULTS AND DISCUSSION}

The results for both cases, linear and nonlinear relative permeabilities curves, will be presented in order to compare the two theories, as well as to show the GITT being applied in solving problems of the petroleum industry.

\subsection{Results for Linear Permeability Curve}

The graphs of Fig. 3 and Fig. 4. Four times are shown for comparing the results found analytically, the saturation differential equation by GITT and the FLUENT solution using the mixture theory. As noted, the numerical results can reproduce the behavior of the saturation front. 


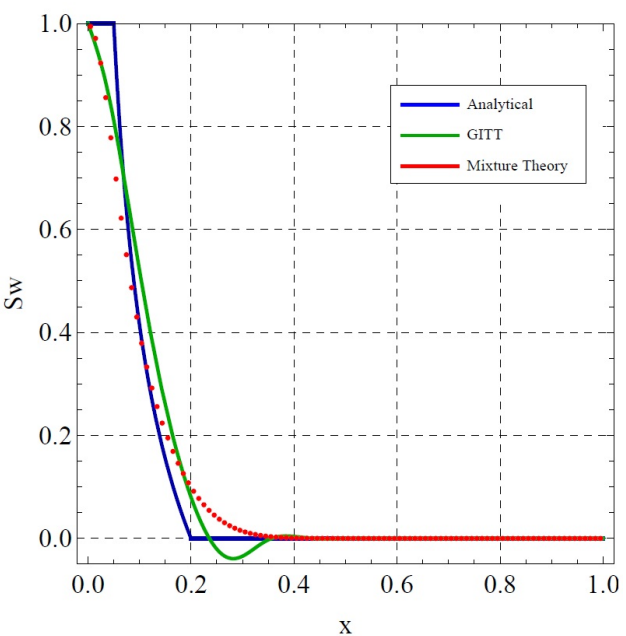

(a) Dimensionless Time $=0.1$

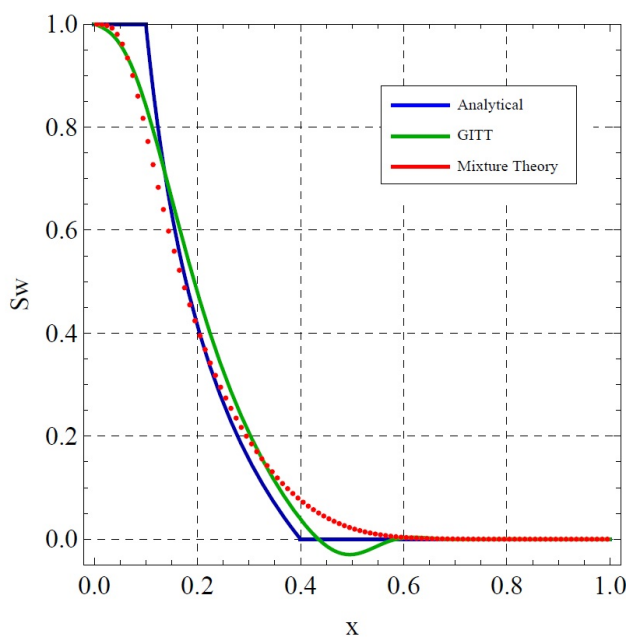

(b) Dimensionless Time $=0.2$

Figure 3. Results for Linear Permeability Curves

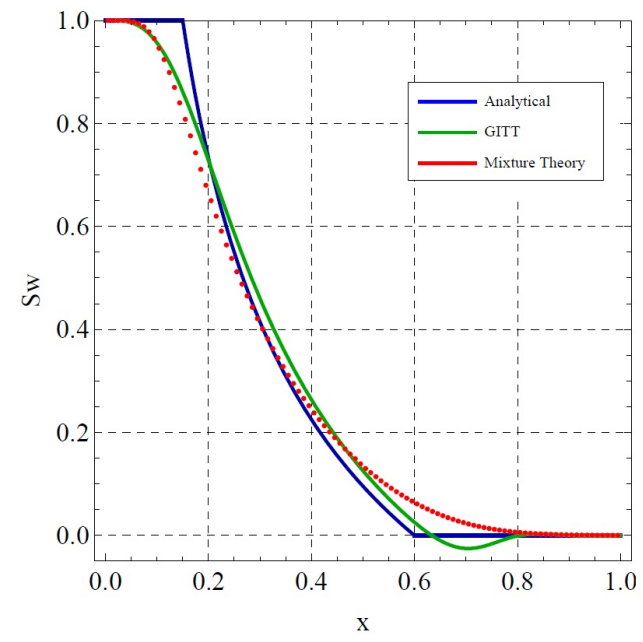

(a) Dimensionless Time $=0.3$

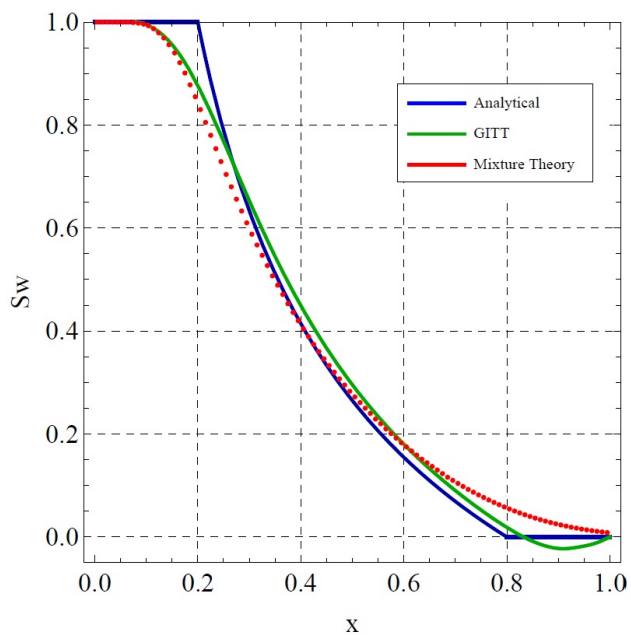

(b) Dimensionless Time $=0.4$

Figure 4. Results for Linear Permeability Curves

Small differences were observed between the coarse meshes and analytical solution. A mesh study was made, but as the paper main objective is to compare the theories, only the best case is presented. All the numerical results converged on the exact solution as the mesh is refined.

The injected water provides two recovery mechanisms: the oil that is ahead of the invaded zone is being pushed in a mechanism similar to a plug flow and in the invaded zone, the oil is being displaced by viscous effects with water. As greater is the saturation shock front saturation, the better will be the displacement efficiency. 


\subsection{Results for Non Linear Permeability Curve}

In Fig. 5 and Fig. 6 can be seen the results for the case of non linear permeability curve shows the the saturation profile along the domain. The same dimensionless four times are shown in order to compare the results found analytically, the saturation differential equation by GITT and finite volumes methods for the theory of mixture.

It can be seen from the results, that the manner in which the water displaces the oil is completely dependent on the type of relative permeability curve.

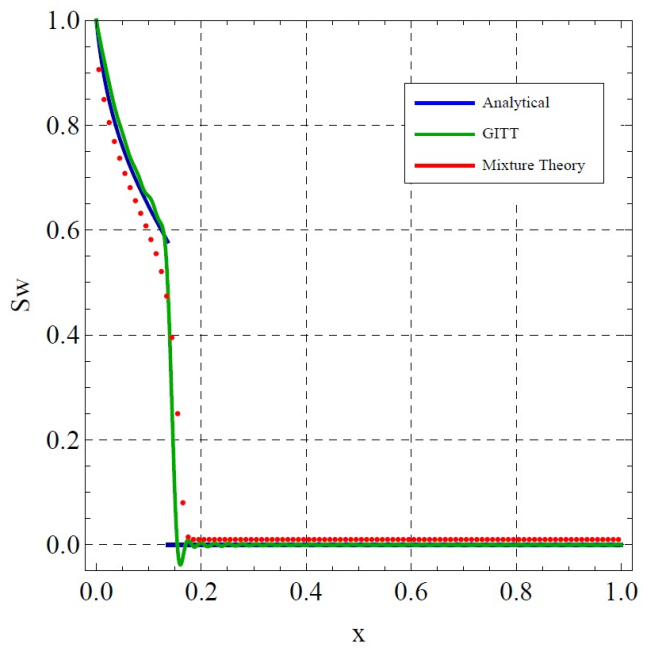

(a) Dimensionless Time $=0.1$

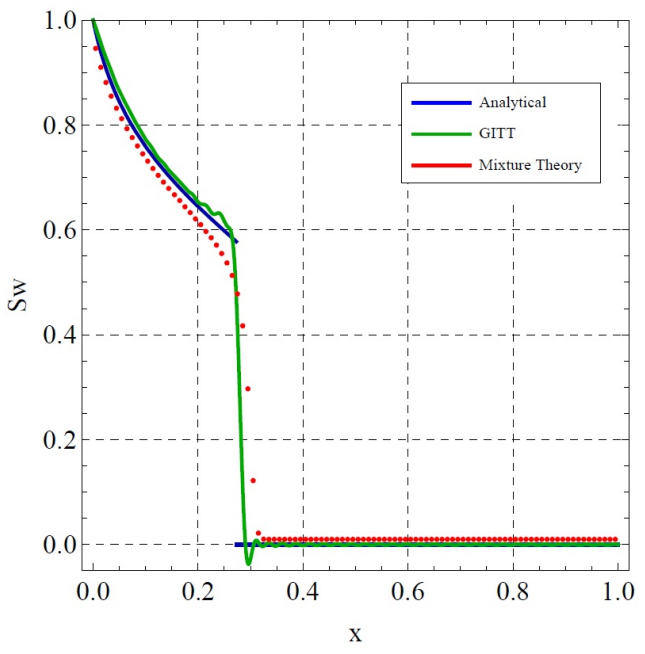

(b) Dimensionless Time $=0.2$

Figure 5. Results for Non Linear Permeability Curves

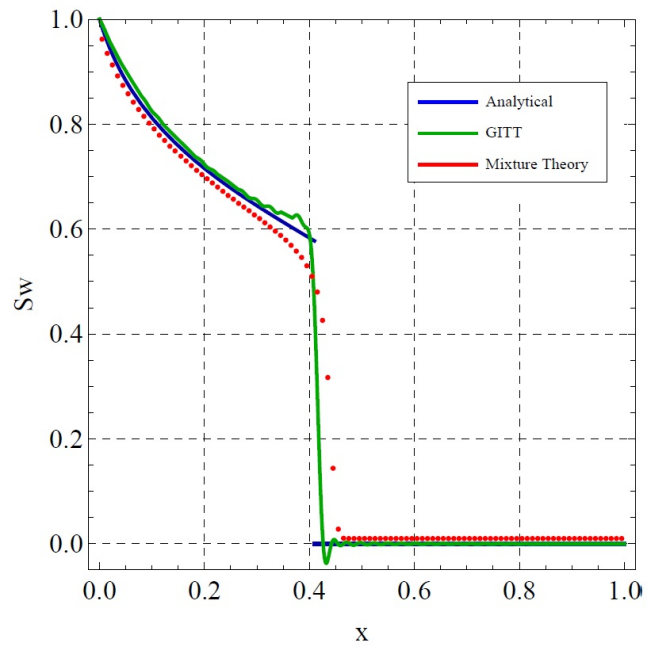

(a) Dimensionless Time $=0.3$

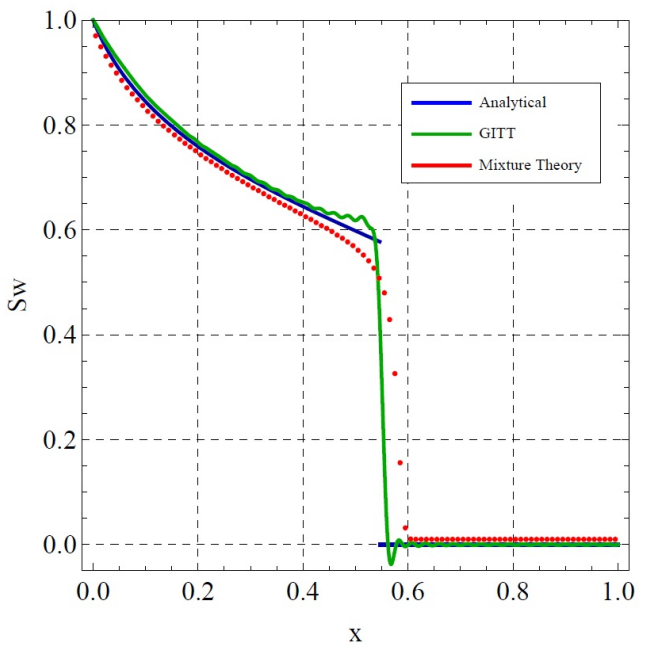

(b) Dimensionless Time $=0.4$

Figure 6. Results for Non Linear Permeability Curves

Just as the linear case, the numerical results can reproduce the behavior of the saturation front. A mesh study also was made for this case and only the best case is presented 
here. For the FLUENT solution using the mixture theory was used a mesh with one hundred elements and for the GITT solution the infinite series was truncated in seventy-five terms.

\section{CONCLUSIONS}

This paper provided a comparison between two different theoretical formulations used in reservoir simulations. The saturation differential equation and the mixture theory were used to solve the recovery of oil by water displacement in a one dimensional case.

The results of both theories are compared with the analytical solution of the best known two phase porous medium equation: the Buckley-Leverett equation. The analytical solution of the Buckley-Leverett equation was obtained following the classical procedures presented in the literature [11, 14].

In order to solve the saturation equation the Generalized Integral Transform Technique was employed. In the case of mixture theory, an advanced, commercial, general-purpose CFD code, FLUENT were used in this work. As noted, the numerical results can reproduce the behavior of the saturation front for both cases.

Finally, in order to study the displacement of oil by water, two relative permeability curves are presented. It can be seen from the results, that the manner in which the water displaces the oil is completely dependent on the type of relative permeability curve. Good results were found for the cases studied.

\section{ACKNOWLEDGEMENTS}

The authors would like to acknowledge the financial support provided by PETROBRAS, ESSS, CNPq, FAPERJ and Universidade Federal Fluminense.

\section{REFERENCES}

\section{References}

[1] J. E. Adkins. Phil. Trans. Roy. Soc. London, A255:607, 1963.

[2] R. J. Atkin and R. E. Craine. Q. J. Mech. Appl. Math., XXIX:209, 1976.

[3] S. E. Buckley and M. C. Leverett. Mechanism of fluid displacement in porous media. Petroleum Technology, pages 107-116, 1942.

[4] J. M. Z. Chongxuan Liu, Jim E. Szecsody and W. P. Ball. Use of the generalized integral transform method for solving equations of solute transport in porous media. Advances in Water Resources, 23(5):483-492, 2000.

[5] R. M. Cotta. Integral Transforms in Computational Heat and Fluid Flow. CRC Press, Boca Raton, FL, 1993.

[6] H. Darcy. Les fountaines publiques de la ville de dijon. Dalmont, Paris, 1856. 
[7] J. R. Fanchi. Principles of Applied Reservoir Simulation. Boston, Gulf Professional Publishing, 1nd edition, 2001.

[8] A. Fick. Ann-der Phys., 94:56, 1976.

[9] A. E. Green and P. M. Naghid. Int. J. Engng. Sci., 3:231, 1965.

[10] A. E. Green and P. M. Naghid. Int. J. Engng. Sci., 6:631, 1968.

[11] R. A. Greenkorn. Flow Phenomena in Porous Media. Marcel Dekker, New York, 1nd edition, 1983.

[12] R. Helmig. Multiphase Flow and Transport Processes in the Subsurface (AContribution to the Modeling of Hydrosystems). Springer, Berlin, Germany, 1nd edition, 1997.

[13] T. S. J.S. Pérez Guerrero a. Analytical solution for one-dimensional advection dispersion transport equation with distance dependent coefficients. Journal of Hydrology, 390:57$65,2010$.

[14] W. Lake, L. Enhanced Oil Recovery. Prentice-Hall, New Jersey, 1nd edition, 1989.

[15] M. L. Martins-Costa and R. M. Saldanha da Gama. A local model for the heat transfer process in two distinct flow regions. Int. J. Heat and Fluid Flow, 15(6), 1994.

[16] D. Nield and A. Bejan. Convection in Porous Media. Springer, New York,, 3nd edition, 2006.

[17] D. W. Peaceman. Fundamentals of Numerical Reservoir Simulation. Elsevier, Englewood Cliffs, NJ, 1977.

[18] L. S. D. A. R. M. Cotta, H. Luznetq and J. N. N. Quaresma. Integral transforms for natural convection in cavities filled with porous media. Transport Phenomena in Porous Media III, (5):97-119, 2000.

[19] R. Sampaio. Arch. Ratl. Mech. Anal., 62:99, 1976.

[20] L. A. Sphaier, R. M. Cotta, C. P. Naveira-Cotta, and J. N. N. Quaresma. The UNIT algorithm for solving one-dimensional convection-diffusion problems via integral transforms. International Communications in Heat and Mass Transfer, 38:565-571, 2011.

[21] C. Truesdell. Rend. Lincei., 22:33, 1957.

[22] C. Truesdell. Rend. Lincei., 22:158, 1957.

[23] C. Truesdell. J. Chem. Phys., 37:2337, 1962.

[24] C. Truesdell and R. Toupin. The Classical Field Theories. Springer-Verlag, New York, NY, 1960. 